\title{
On Chow Quotients of Torus Actions
}

\author{
HeNdrik BäKER, JÜRgen HaUSEN, \& Simon KeICHER
}

\begin{abstract}
We consider torus actions on Mori dream spaces and ask whether the associated Chow quotient is again a Mori dream space and, if so, what does its Cox ring look like. We provide general tools for the study of these problems and give solutions for $\mathbb{K}^{*}$-actions on smooth quadrics.
\end{abstract}

\section{Introduction}

Consider an action $G \times X \rightarrow X$ of a connected linear algebraic group $G$ on a projective variety $X$ defined over an algebraically closed field $\mathbb{K}$ of characteristic zero. The Chow quotient is an answer to the problem of associating in a canonical way a quotient to this action: it is defined as the closure of the set of general $G$-orbit closures viewed as points in the Chow variety; see Section 2 for more background. The Chow quotient always exists, but, in general, its geometry appears to be not easily accessible.

In the present paper, we consider algebraic torus actions $T \times X \rightarrow X$ and ask for the Mori dream property of the normalized Chow quotient $Y$, provided that $X$ is a Mori dream space, that is, has finitely generated Cox ring [14]. A well understood example class is given by subtorus actions on toric varieties. There, the normalized Chow quotient is again toric and hence a Mori dream space. Moreover, the corresponding fan can be computed, and thus the Cox ring of the normalized Chow quotient is accessible as well $[16 ; 6]$. Note, however, that there is no hope for comparable statements in general. For example, Castravet and Tevelev [5] showed that the Chow quotient $\bar{M}_{0, n}$ of the maximal torus action on the Grassmannian $G(2, n)$ is not a Mori dream space for $n$ sufficiently large.

Our aim is to provide tools for the treatment of nontoric examples and to open up the case of $\mathbb{K}^{*}$-actions on smooth projective quadrics as a new example class for positive results. The first main result is the following.

TheOREM 1.1. Let $\mathbb{K}^{*}$ act on a smooth projective quadric X. Then the associated normalized Chow quotient is a Mori dream space.

The second result concerns the computation of the Cox ring; recall that the explicit knowledge of the Cox ring is an approach to the geometry of the underlying space [1]. We first prepare and state the result and then discuss the setting. After an equivariant embedding into a projective space and applying a suitable linear

Received August 23, 2013. Revision received May 19, 2015. 
transformation, any smooth projective quadric $X$ is of the following shape:

$$
X=V\left(g_{1}\right) \subseteq \mathbb{P}_{r}, \quad g_{1}= \begin{cases}T_{0} T_{1}+\cdots+T_{r-1} T_{r}, & r \text { odd }, \\ T_{0} T_{1}+\cdots+T_{r-2} T_{r-1}+T_{r}^{2}, & r \text { even }\end{cases}
$$

where the $\mathbb{K}^{*}$-action is diagonal with weights $\zeta_{0}, \ldots, \zeta_{r}$, and the defining equation is of degree zero. In order to write down the Cox ring of the Chow quotient, consider the extended weight matrix

$$
Q:=\left[\begin{array}{ccc}
\zeta_{0} & \ldots & \zeta_{r} \\
1 & \ldots & 1
\end{array}\right]
$$

where we assume that the columns of $Q$ generate $\mathbb{Z}^{2}$. Let $P$ be an integral Gale dual, that is, an $r-1$ by $r+1$ matrix with the row space of $Q$ as kernel. Determine the Gelfand-Kapranov-Zelevinsky decomposition $\Sigma$ associated to $P$ and put the primitive generators $b_{1}, \ldots, b_{l}$ of $\Sigma$ differing from the columns of $P$ as columns into a matrix $B$. Then there is an integral matrix $A$ such that $B=P \cdot A$. Define the shifted row sums

$$
\eta_{i}:=A_{i *}+A_{i+1 *}+\mu \quad \text { for } i=0,2, \ldots \quad \text { and } \quad \eta_{r}:=2 A_{r *}+\mu \quad \text { if } r \text { is even, }
$$

where $\mu$ is the componentwise minimal vector such that the entries of the $\eta_{i}$ are all nonnegative. Then our result reads as follows.

THEOREM 1.2. In the previous setting, assume that any $r$ columns of $Q$ generate $\mathbb{Z}^{2}$, there remain at least two different weights $\zeta_{i}$ when removing two of maximal absolute value, and for odd (even) $r$, there are at least four (three) $\zeta_{i}$ of minimal absolute value. Then the normalized Chow quotient $Y$ of the $\mathbb{K}^{*}$-action on $X$ has the Cox ring

$$
\mathcal{R}(Y)=\mathbb{K}\left[T_{0}, \ldots, T_{r}, S_{1}, \ldots, S_{l}\right] /\left\langle g_{2}\right\rangle
$$

with

$$
g_{2}:= \begin{cases}T_{0} T_{1} S^{\eta_{0}}+T_{2} T_{3} S^{\eta_{2}}+\cdots+T_{r-1} T_{r} S^{\eta_{r-1}}, & r \text { odd }, \\ T_{0} T_{1} S^{\eta_{0}}+\cdots+T_{r-2} T_{r-1} S^{\eta_{r-2}}+T_{r}^{2} S^{\eta_{r}}, & r \text { even },\end{cases}
$$

graded by $\mathbb{Z}^{l+2}$ via assigning to the ith variable the ith column of a Gale dual of the block matrix $[P, B]$.

Let us shed some light on this setting. The assumptions that any $r$ columns of $Q$ generate $\mathbb{Z}^{2}$ and there remain at least two different weights $\zeta_{i}$ when removing two of maximal absolute value mean exactly that $Q$ defines a Cox ring of a projective "intrinsic quadric" $Y^{\prime}$ in the sense of [3] with divisor class group $\mathbb{Z}^{2}$; see [4] for other research on such varieties. The meaning of the Chow quotient $Y$ computed in Theorem 1.2 is that it dominates in a minimal manner all normal projective varieties $Y^{\prime \prime}$ allowing a small quasi-modification $Y^{\prime \prime} \rightarrow Y^{\prime}$; see also Remark 4.6. A discussion of the assumption that, for odd (even) $r$, there are at least four (three) $\zeta_{i}$ of minimal absolute value is given in Remark 4.7. 
The proof of Theorem 1.2 is performed in Section 4. Besides the explicit description of the rays of the Gelfand-Kapranov-Zelevinsky decomposition provided in Proposition 4.1, it requires controlling the behavior of the Cox ring under certain modifications. This technique is of independent interest and developed in full generality in Section 3. The proof of Theorem 1.1, given in Section 5, uses moreover methods from tropical geometry: we consider a "weak tropical resolution" of the Chow quotient (see Construction 5.3) and provide a reduction principle to divide out intrinsic torus symmetry (see Proposition 5.6).

\section{Chow Quotients and Limit Quotients}

We present the necessary background and a general result for the action of a torus $T$ on a projective (irreducible) variety $X$. For a precise definition of the quotients, consider more generally the action $G \times X \rightarrow X$ of any connected linear algebraic group $G$ on a projective variety $X$. The Chow quotient has been introduced by Kapranov, Sturmfels, and Zelevinsky [16]. Initially, the construction appears to depend on an embedding but finally turns out not to do so.

Construction 2.1. Suppose that $X$ is a $G$-invariant closed subvariety of some projective space. For a suitable open invariant subset $U \subseteq X$, all orbit closures $c(x):=\overline{G \cdot x}$, where $x \in U$, have the same dimension $k$ and degree $d$. Thus, each $x \in U$ defines a point $c(x) \in \mathrm{Ch}(X)$ in the Chow variety of $k$-cycles of degree $d$. The Chow quotient of the $G$-action on $X$ is the closure

$$
X_{\text {ce }}^{\prime} G:=\overline{\{c(x) ; x \in U\}} \subseteq \mathrm{Ch}(X) .
$$

By the normalized Chow quotient we mean the normalization $X_{\mathrm{cQ}}^{\tilde{I}} G$ of $X_{\mathrm{cQ}}^{\prime} G$. With a suitably small chosen $U \subseteq X$, we obtain a commutative diagram of morphisms involving the normalization map:

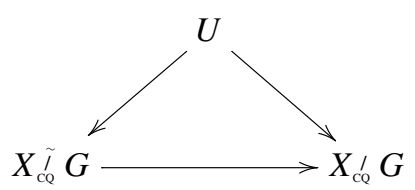

The limit quotient arises from the variation of Mumford's GIT quotients [19]. Its construction relies on finiteness of the number of possible sets of semistable points [8;21].

Construction 2.2. Suppose that $G$ is reductive. Let $X_{1}, \ldots, X_{r} \subseteq X$ be open sets of semistable points arising from $G$-linearized ample line bundles on $X$. Then, whenever $X_{i} \subseteq X_{j}$, we have a commutative diagram

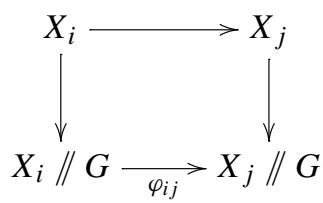


where the induced map $\varphi_{i j}: X_{i} / / G \rightarrow X_{j} / / G$ of quotients is a dominant projective morphism. This turns the quotient spaces into a directed system, the GIT system. The associated GIT limit $Y$, that is, the inverse limit, comes with a canonical morphism

$$
U:=\bigcap_{i=1}^{r} X_{i} \rightarrow Y .
$$

The closure of the image of this morphism is denoted by $X_{\llcorner Q}^{\prime} G$ and is called the limit quotient. There are canonical proper birational morphisms onto the GIT quotients:

$$
\pi_{i}: X_{\mathrm{LQ}}^{\prime} G \rightarrow X_{i} / / G
$$

The normalized limit quotient is the normalization $X_{\mathrm{LQ}}^{\tilde{I}} G$ of $X_{\mathrm{LQ}}^{\prime} G$. Suitably shrinking the open set $U \subseteq X$, we obtain a commutative diagram involving the normalization map:

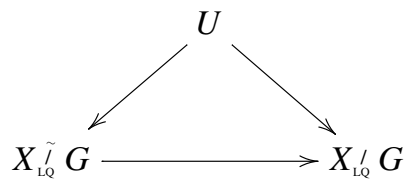

Note that, in the literature, $X_{\mathrm{LQ}}^{\prime} G$ is also called the "canonical component" of the GIT limit, or even shortly the "GIT limit". Similar to the full inverse limit, the quotient $X_{\mathrm{LQ}}^{\prime} G$ enjoys a universal property.

REMARK 2.3. Given an irreducible variety $W$ and a collection of dominant morphisms $\psi_{i}: W \rightarrow X_{i} / / G$ with $\psi_{j}=\varphi_{i j} \circ \psi_{i}$ for all $i, j$, there is a unique morphism $\psi: W \rightarrow X_{\mathrm{LQ}}^{\prime} G$ with $\psi_{i}=\pi_{i} \circ \psi$ for all $i$.

For a general reductive group action, the (normalized) Chow quotient and the (normalized) limit quotient need not coincide. For torus actions, however, they do. This statement seems to have folklore status; a proof under a certain hypothesis can be found in [13, Thm. 3.8]. Let us indicate how to deduce it from the corresponding statement in the case of subtorus actions on projective toric varieties obtained in $[16 ; 6]$.

We recall the necessary results and concepts from [16; 6]. Let $Z$ be a projective toric variety with acting torus $T_{Z}$ and consider the action of a subtorus $T \subseteq T_{Z}$. The toric variety $Z$ arises from a fan $\Sigma$ in some $\mathbb{Z}^{r}$, and $T \subseteq T_{Z}$ corresponds to an embedding $\mathbb{Z}^{k} \subseteq \mathbb{Z}^{r}$ of a sublattice. Let $P: \mathbb{Z}^{r} \rightarrow \mathbb{Z}^{r-k}$ be the projection. The quotient fan of $\Sigma$ with respect to $P$ is the fan in $\mathbb{Z}^{r-k}$ with the cones

$$
\tau(v):=\bigcap_{\sigma \in \Sigma, v \in P(\sigma)} P(\sigma), \quad v \in \mathbb{Q}^{r-k} .
$$

Proposition 2.4. See $[16 ; 6]$. Consider the toric variety $Z$ arising from a fan $\Sigma$ in $\mathbb{Z}^{r}$ and the action of a subtorus $T \subseteq T_{Z}$ corresponding to a sublattice $\mathbb{Z}^{k} \subseteq$ $\mathbb{Z}^{r}$. Let $\Sigma^{\prime}$ be the quotient fan in $\mathbb{Z}^{r-\bar{k}}$ with respect to $\mathbb{Z}^{r} \rightarrow \mathbb{Z}^{r-k}$, and $Z^{\prime}$ the 
associated toric variety. Then we have a commutative diagram

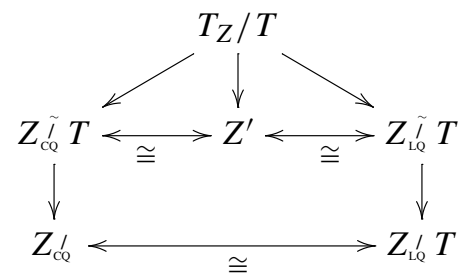

In particular, the (normalized) Chow quotient and the (normalized) limit quotient of the $T$-action on $Z$ are isomorphic to each other.

We turn to the general case. The result is formulated for a projective variety $X$ that is equivariantly embedded into a toric variety $Z$. Note that for a normal projective $X$, this can always be achieved, even with a projective space $Z$.

Proposition 2.5. Let $Z$ be a projective toric variety, $T \subseteq T_{Z}$ a subtorus of the big torus, and $X \subseteq Z$ a closed $T$-invariant subvariety intersecting $T_{Z}$. Then there is a commutative diagram

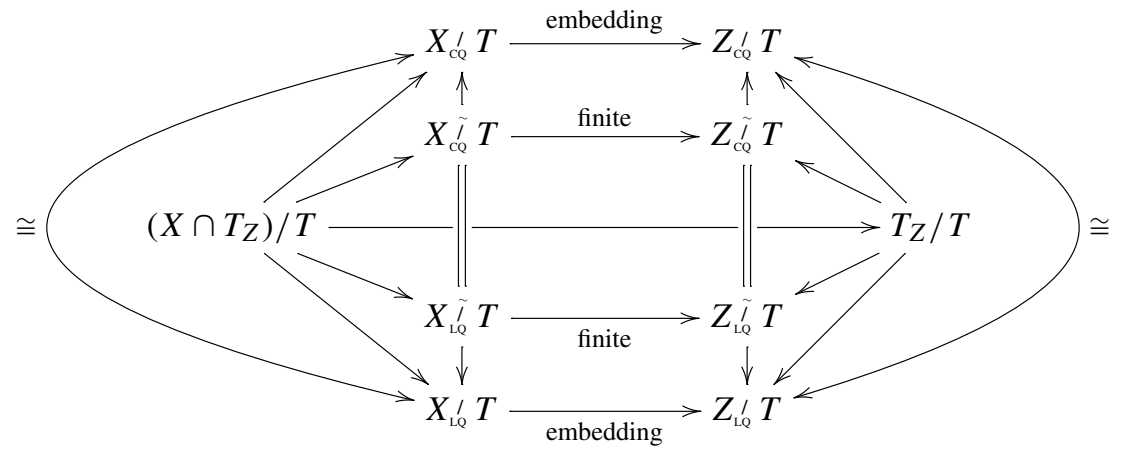

where $X_{\mathrm{cQ}}^{\tilde{i}} T \rightarrow Z_{\mathrm{cQ}}^{\tilde{i}} T$ and $X_{\mathrm{LQ}}^{\tilde{I}} T \rightarrow Z_{\mathrm{L}}^{\tilde{i}} T$ normalize the closures of the images of $\left(X \cap T_{Z}\right) / T$ under the canonical open embeddings of $T_{Z} / T$.

Proof. The right part of the diagram is Proposition 2.4. The closed embedding $X_{\mathrm{cQ}}^{\prime} T \rightarrow Z_{\mathrm{cQ}}^{\prime} T$ exists by the construction of the Chow quotient; compare also [9, Thm. 3.2].

To obtain a morphism $X_{\mathrm{LQ}}^{\prime} T \rightarrow Z_{\mathrm{LQ}}^{\prime} T$, consider the sets of semistable points $V_{1}, \ldots, V_{s} \subseteq Z_{\text {ce }}^{\tilde{i}} T$ defined by $T$-linearized ample line bundles on $Z$. Then the sets $U_{i}:=X \cap V_{i}$ are sets of semistable points of the respective pullback bundles, see [19, Thm. 1.19], and we have induced morphisms $U_{i} / / T \rightarrow V_{i} / / T$. Since the $U_{i} / / T$ form a subsystem of the full GIT-system of $X$, the universal property (Remark 2.3) yields a morphism of the limit quotients sending $X_{\mathrm{LQ}}^{\prime} T$ birationally onto the closure of $\left(X \cap T_{Z}\right) / T$.

Now look at the canonical morphism $X_{\mathrm{cQ}}^{\prime} T \rightarrow X_{\mathrm{LQ}}^{\prime} T$ provided by [15;21]. It fits into the diagram established so far, which in turn implies that $X_{\mathrm{CQ}}^{\prime} T \rightarrow X_{\mathrm{LQ}}^{\prime} T$ 
is an isomorphism and $X_{\mathrm{LQ}}^{\prime} T \rightarrow Z_{\mathrm{LQ}}^{\prime} T$ is an embedding. Finally, the respective normalizations fit into the diagram via their universal properties.

Note that we will only use the part of Proposition 2.5 concerning the normalizations. This can be proved by similar arguments as before but without using the isomorphism $Z_{\mathrm{CQ}}^{\prime} T \rightarrow Z_{\mathrm{LQ}}^{\prime} T$ of Proposition 2.4.

Corollary 2.6. Let $T \times X \rightarrow X$ be the action of a torus $T$ on a normal projective variety $X$. Then the normalized Chow quotient $X_{\mathrm{cQ}}^{\tilde{I}} T$ and the normalized limit quotient $X_{\mathrm{LQ}}^{\tilde{I}} T$ are isomorphic to each other.

The following corollary shows that for torus actions, the limit quotient is up to normalization already determined by the possible linearizations of a single ample bundle, a statement that fails in general for other reductive groups; compare also [15, Rem. 0.4.10].

Corollary 2.7. Let $T \times X \rightarrow X$ be the action of a torus $T$ on a normal projective variety $X$. Then the subsystem of GIT quotients arising from the possible $T$-linearizations of a given ample line bundle $\mathcal{L}$ has the same normalized limit quotient as the full system of GIT quotients.

Proof. Fix a $T$-linearization of $\mathcal{L}$ and consider the $T$-equivariant embedding $X \rightarrow \mathbb{P}_{r}$ defined by a suitable power of $\mathcal{L}$. Then the subsystem of the GIT quotients on $X$ arising from other linearizations of $\mathcal{L}$ is induced from the full GIT system on $\mathbb{P}_{r}$. Now apply Proposition 2.5 .

We now prove a reduction theorem, which says in particular that the Chow quotient of a torus action is birationally dominated by an iterated Chow quotient with respect to $\mathbb{K}^{*}$-actions.

Theorem 2.8. Let $T \times X \rightarrow X$ be the action of a torus $T$ on a normal projective variety $X$. Fix a subtorus $T_{0} \subseteq T$ and set $T_{1}:=T / T_{0}$. Then we have canonical proper birational morphisms

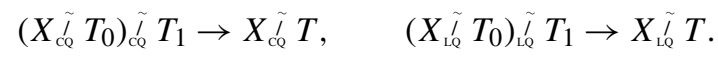

Proof. First, consider the case that $T$ is a subtorus of the big torus $T_{Z}$ of a toric variety $Z$. Then the maps $T_{Z} \rightarrow T_{Z} / T_{0} \rightarrow T_{Z} / T$ correspond to lattice homomorphisms $\mathbb{Z}^{r} \rightarrow \mathbb{Z}^{r-k_{0}} \rightarrow \mathbb{Z}^{r-k}$. The fan $\Sigma$ of $Z$ lives in $\mathbb{Z}^{r}$, and we have the quotient fan $\Sigma_{0}$ of $\Sigma$ with respect to $\mathbb{Z}^{r} \rightarrow \mathbb{Z}^{r-k_{0}}$. The quotient fan of $\Sigma_{0}$ with respect to $\mathbb{Z}^{r-k_{0}} \rightarrow \mathbb{Z}^{r-k}$ refines the quotient fan of $\Sigma$ with respect to $\mathbb{Z}^{r} \rightarrow \mathbb{Z}^{r-k}$. Translated to toric varieties, this means that we have the desired maps

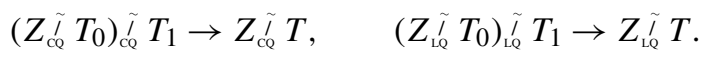

We turn to the general case. Suitably embedding $X$, we can arrange the setup of Proposition 2.5. Then we have a finite $T_{1}$-equivariant map $v: X_{\mathrm{cQ}}^{\tilde{l}} T_{0} \rightarrow Z_{\mathrm{cQ}}^{\tilde{l}} T_{0}$. We consider the normalized limit quotient of the $T_{1}$-action on $X_{\mathrm{c}}^{\tilde{c}} T_{0}$. In a first 
step, we establish a commutative diagram

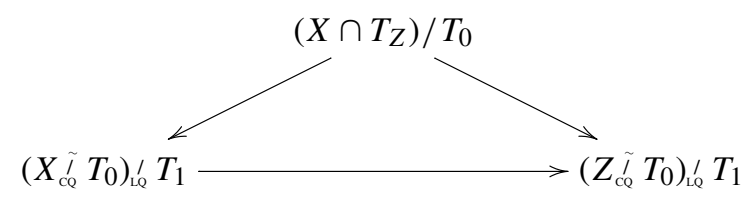

For this, let $V_{1}, \ldots, V_{s} \subseteq Z_{\mathrm{cQ}}^{\tilde{i}} T_{0}$ be the sets of semistable points arising from $T_{1}$ linearized ample line bundles. Then the inverse images $v^{-1}\left(V_{i}\right) \subseteq X_{\text {co }}^{\tilde{I}} T_{0}$ are sets of semistable points of the respective pullback bundles, see [19, Thm. 1.19]. Note that we have canonical induced maps

$$
v^{-1}\left(V_{i}\right) / / T_{1} \rightarrow V_{i} / / T_{1}
$$

Consequently, the limit quotient of the system of the quotients $v^{-1}\left(V_{i}\right) / / T_{1}$ maps to the limit quotient $\left(Z_{\mathrm{CQ}}^{\tilde{\gamma}} T_{0}\right)_{\mathrm{LQ}} T_{1}$. Since the $v^{-1}\left(V_{i}\right) / / T_{1}$ form a subsystem of the full GIT system of $X_{\mathrm{cQ}}^{\tilde{i}} T_{0}$, this gives rise to a morphism

$$
\left(X_{\mathrm{CQ}}^{\tilde{l}} T_{0}\right)_{\mathrm{LQ}}^{\prime} T_{1} \rightarrow\left(Z_{\mathrm{cQ}}^{\tilde{\jmath}} T_{0}\right)_{\mathrm{LQ}}^{\prime} T_{1}
$$

as needed for the previous commutative diagram. As in the proof of Proposition 2.5, we may pass to the normalizations and thus obtain a morphism

$$
\left(X_{\mathrm{cQ}}^{\tilde{j}} T_{0}\right)_{\mathrm{cQ}}^{\tilde{i}} T_{1} \rightarrow\left(Z_{\mathrm{CQ}}^{\tilde{I}} T_{0}\right)_{\mathrm{cQ}}^{\tilde{I}} T_{1}
$$

Now, by the toric case, we have a proper birational morphism from the toric variety on the right-hand side onto $Z_{\mathrm{cQ}}^{\tilde{I}} T$. Using once more Proposition 2.5, the assertion follows.

\section{Toric Ambient Modifications}

In this section, we provide a general machinery to study the effect of modifications on the Cox ring. Similar to [17], we use toric embeddings. In contrast to the geometric criteria given there, our approach here is purely algebraic, based on the results of [2]. The heart is a construction of factorially graded rings out of given ones.

We begin with recalling the necessary algebraic concepts. Let $K$ be a finitely generated Abelian group, and $R$ a finitely generated integral $K$-graded $\mathbb{K}$-algebra. A homogeneous nonzero nonunit $f \in R$ is called $K$-prime if $f \mid g h$ with homogeneous $g, h \in R$ always implies $f \mid g$ or $f \mid h$. The algebra $R$ is called factorially $K$-graded if every homogeneous nonzero nonunit $f \in R$ is a product of $K$-primes.

We enter the construction of factorially graded rings. Consider a grading of the polynomial ring $\mathbb{K}\left[T_{1}, \ldots, T_{r_{1}}\right]$ by a finitely generated Abelian group $K_{1}$ such 
that the variables $T_{i}$ are homogeneous. Then we have a pair of exact sequences

$$
\begin{aligned}
& 0 \longrightarrow \mathbb{Z}^{k_{1}} \stackrel{Q_{1}^{*}}{\longrightarrow} \mathbb{Z}^{r_{1}} \stackrel{P_{1}}{\longrightarrow} \mathbb{Z}^{n} \\
& 0 \lessdot K_{1} \lessdot{ }_{Q_{1}} \mathbb{Z}^{r_{1}} \leftarrow_{P_{1}^{*}} \mathbb{Z}^{n} \leftarrow 0
\end{aligned}
$$

where $Q_{1}: \mathbb{Z}^{r_{1}} \rightarrow K_{1}$ is the degree map sending the $i$ th canonical basis vector $e_{i}$ to $\operatorname{deg}\left(T_{i}\right) \in K_{1}$. We enlarge $P_{1}$ to an $n \times r_{2}$ matrix $P_{2}$ by concatenating further $r_{2}-r_{1}$ columns. This gives a new pair of exact sequences

$$
\begin{aligned}
& 0 \longrightarrow \mathbb{Z}^{k_{2}} \stackrel{Q_{2}^{*}}{\longrightarrow} \mathbb{Z}^{r_{2}} \stackrel{P_{2}}{\longrightarrow} \mathbb{Z}^{n} \\
& 0 \lessdot K_{2} \leftarrow{ }_{Q_{2}} \mathbb{Z}^{r_{2}} \leftarrow_{P_{2}^{*}} \mathbb{Z}^{n} \leftarrow 0
\end{aligned}
$$

Construction 3.1. Given a $K_{1}$-homogeneous ideal $I_{1} \subseteq \mathbb{K}\left[T_{1}, \ldots, T_{r_{1}}\right]$, we transfer it to a $K_{2}$-homogeneous ideal $I_{2} \subseteq \mathbb{K}\left[T_{1}, \ldots, T_{r_{2}}\right]$ by taking extensions and contractions according to the scheme

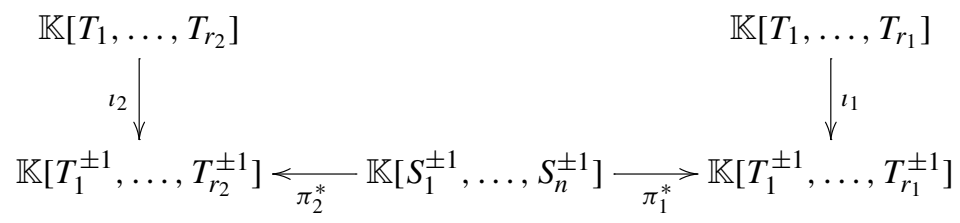

where $\iota_{1}, l_{2}$ are the canonical embeddings, and $\pi_{i}^{*}$ are the homomorphisms of group algebras defined by $P_{i}^{*}: \mathbb{Z}^{n} \rightarrow \mathbb{Z}^{r_{i}}$.

Now let $I_{1} \subseteq \mathbb{K}\left[T_{1}, \ldots, T_{r_{1}}\right]$ be a $K_{1}$-homogeneous ideal, and $I_{2} \subseteq \mathbb{K}\left[T_{1}, \ldots, T_{r_{2}}\right]$ the transferred $K_{2}$-homogeneous ideal. Our result relates factoriality properties of the algebras $R_{1}:=\mathbb{K}\left[T_{1}, \ldots, T_{r_{1}}\right] / I_{1}$ and $R_{2}:=\mathbb{K}\left[T_{1}, \ldots, T_{r_{2}}\right] / I_{2}$ to each other.

Theorem 3.2. Assume that $R_{1}, R_{2}$ are integral, $T_{1}, \ldots, T_{r_{1}}$ define $K_{1}$-primes in $R_{1}$, and $T_{1}, \ldots, T_{r_{2}}$ define $K_{2}$-primes in $R_{2}$. Then the following statements are equivalent:

(i) The algebra $R_{1}$ is factorially $K_{1}$-graded.

(ii) The algebra $R_{2}$ is factorially $K_{2}$-graded. 
Proof. First, observe that the homomorphisms $\pi_{j}^{*}$ embed $\mathbb{K}\left[S_{1}^{ \pm 1}, \ldots, S_{n}^{ \pm 1}\right]$ as the degree zero part of the respective $K_{j}$-grading and fit into a commutative diagram

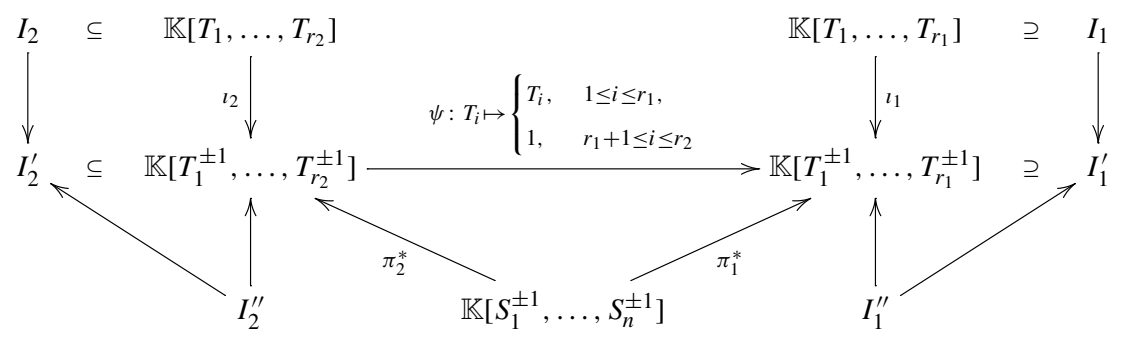

The factor ring $R_{1}^{\prime}$ of the extension $I_{1}^{\prime}:=\left\langle l_{1}\left(I_{1}\right)\right\rangle$ is obtained from $R_{1}$ by localization with respect to $K_{1}$-primes $T_{1}, \ldots, T_{r_{1}}$ :

$$
R_{1}^{\prime}:=\mathbb{K}\left[T_{1}^{ \pm 1}, \ldots, T_{r_{1}}^{ \pm 1}\right] / I_{1}^{\prime} \cong\left(R_{1}\right)_{T_{1} \cdots T_{r_{1}}} .
$$

The ideal $I_{1}^{\prime \prime}$ is the degree zero part of $I_{1}^{\prime}$. Thus, its factor algebra is the degree zero part of $R_{1}^{\prime}$ :

$$
R_{1}^{\prime \prime}:=\mathbb{K}\left[T_{1}^{ \pm 1}, \ldots, T_{r_{1}}^{ \pm 1}\right]_{0} / I_{1}^{\prime \prime} \cong\left(R_{1}^{\prime}\right)_{0} .
$$

Note that $\mathbb{K}\left[T_{1}^{ \pm 1}, \ldots, T_{r_{1}}^{ \pm 1}\right]$ and hence $R_{1}^{\prime}$ admit units in every degree. Thus, [2, Thm. 1.1] yields that $R_{1}$ is a factorially $K_{1}$-graded if and only if $R_{1}^{\prime \prime}$ is a UFD.

The homomorphism $\psi$ restricts to an isomorphism $\psi_{0}$ of the respective degree zero parts. Thus, the shifted ideal $I_{2}^{\prime \prime}:=\psi_{0}^{-1}\left(I_{1}^{\prime \prime}\right)$ defines an algebra $R_{2}^{\prime \prime}$ isomorphic to $R_{1}^{\prime \prime}$ :

$$
R_{2}^{\prime \prime}:=\mathbb{K}\left[T_{1}^{ \pm 1}, \ldots, T_{r_{2}}^{ \pm 1}\right]_{0} / I_{2}^{\prime \prime} \cong R_{1}^{\prime \prime} .
$$

The ideal $I_{2}^{\prime}:=\left\langle\pi_{2}^{*}\left(\left(\pi_{1}^{*}\right)^{-1}\left(I_{1}^{\prime}\right)\right)\right\rangle$ has $I_{2}^{\prime \prime}$ as its degree zero part, and $\mathbb{K}\left[T_{1}^{ \pm 1}, \ldots\right.$, $T_{r_{2}}^{ \pm 1}$ ] admits units in every degree. The associated $K_{2}$-graded algebra

$$
R_{2}^{\prime}:=\mathbb{K}\left[T_{1}^{ \pm 1}, \ldots, T_{r_{2}}^{ \pm 1}\right] / I_{2}^{\prime}
$$

is the localization of $R_{2}$ by the $K_{2}$-primes $T_{1}, \ldots, T_{r_{2}}$. Again by [2, Thm. 1.1] we obtain that $R_{2}^{\prime \prime}$ is a UFD if and only if $R_{2}$ is factorially $K_{2}$-graded.

The following observation is intended for practical purposes; it reduces, for example, the number of necessary primality tests.

Proposition 3.3. Assume that $R_{1}$ is integral and the canonical map $K_{2} \rightarrow K_{1}$ admits a section (e.g., $K_{1}$ is free).

(i) Let $T_{1}, \ldots, T_{r_{1}}$ define $K_{1}$-primes in $R_{1}$ and $T_{r_{1}+1}, \ldots, T_{r_{2}}$ define $K_{2}$-primes in $R_{2}$. If no $T_{j}$ with $j \geq r_{1}+1$ divides a $T_{i}$ with $i \leq r_{1}$, then also $T_{1}, \ldots, T_{r_{1}}$ define $K_{2}$-primes in $R_{2}$.

(ii) The ring $R_{2}$ is integral. Moreover, if $R_{1}$ is normal and $T_{r_{1}+1}, \ldots, T_{r_{2}}$ define primes in $R_{2}$ (e.g., they are $K_{2}$-prime, and $K_{2}$ is free), then $R_{2}$ is normal. 
Proof. The exact sequences involving the grading groups $K_{1}$ and $K_{2}$ fit into a commutative diagram where the upwards sequences are exact and $\mathbb{Z}^{r_{2}-r_{1}} \rightarrow K_{2}^{\prime}$ is an isomorphism:

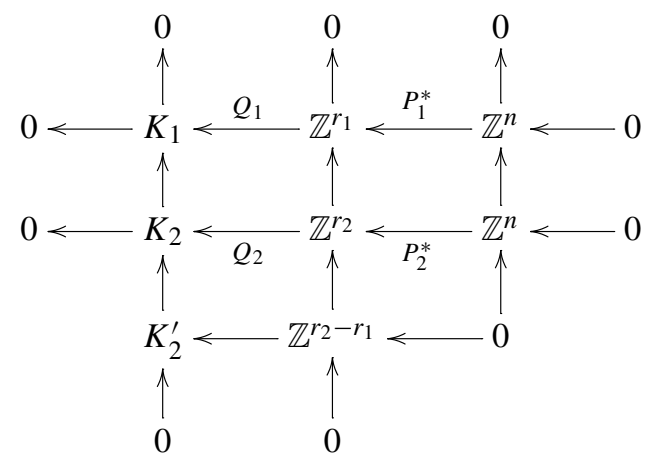

Moreover, denoting by $K_{1}^{\prime} \subseteq K_{2}$ the image of the section $K_{1} \rightarrow K_{2}$, there is a splitting $K_{2}=K_{2}^{\prime} \oplus K_{1}^{\prime}$. Since $K_{2}^{\prime} \subseteq K_{2}$ is the subgroup generated by the degrees of $T_{r_{1}+1}, \ldots, T_{r_{2}}$, we obtain a commutative diagram

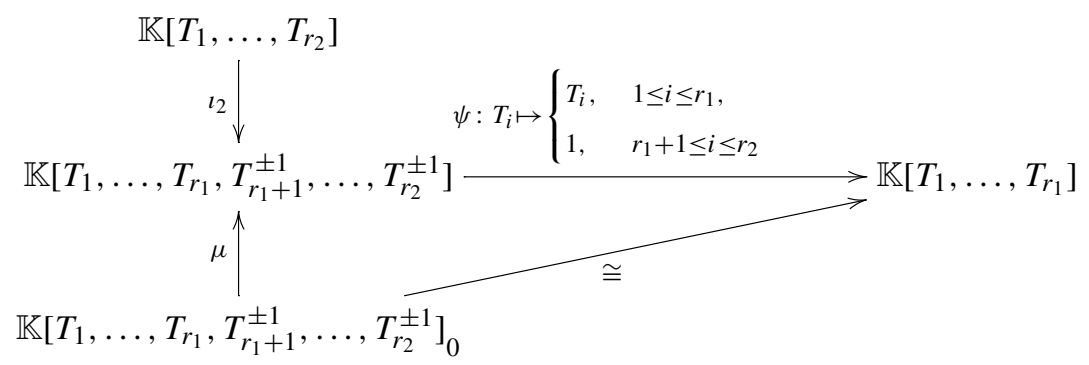

where the map $\mu$ denotes the embedding of the degree zero part with respect to the $K_{2}^{\prime}$-grading. By the splitting $K_{2}=K_{2}^{\prime} \oplus K_{1}^{\prime}$ the image of $\mu$ is precisely the Veronese subalgebra associated with the subgroup $K_{1}^{\prime} \subseteq K_{2}$. For the factor rings $R_{2}$ and $R_{1}$ by the ideals $I_{2}$ and $I_{1}$, the previous diagram leads to the following situation:

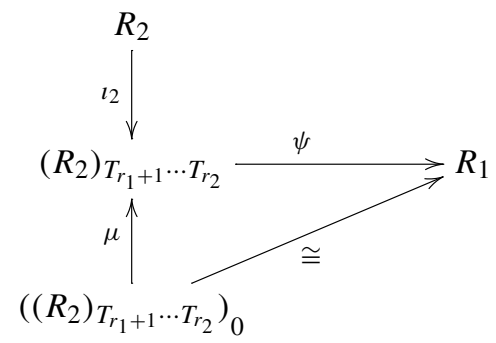

To prove (i), consider a variable $T_{i}$ with $1 \leq i \leq r_{1}$. We have to show that $T_{i}$ defines a $K_{2}$-prime element in $R_{2}$. By the previous diagram, $T_{i}$ defines a $K_{1}^{\prime}$ prime element in $\left(\left(R_{2}\right)_{T_{r_{1}+1} \cdots T_{r_{2}}}\right)_{0}$, the Veronese subalgebra of $R_{2}$ defined by $K_{1}^{\prime} \subseteq K_{2}$. Since every $K_{2}$-homogeneous element of $\left(R_{2}\right)_{T_{r_{1}+1} \cdots T_{r_{2}}}$ can be shifted 
by a homogeneous unit into $\left(\left(R_{2}\right)_{T_{r_{1}+1} \cdots T_{r_{2}}}\right)_{0}$, we see that $T_{i}$ defines a $K_{2}$-prime in $\left(R_{2}\right)_{T_{r_{1}+1} \cdots T_{r_{2}}}$. By assumption, $T_{r_{1}+1}, \ldots, T_{r_{2}}$ define $K_{2}$-primes in $R_{2}$ and are all coprime to $T_{i}$. It follows that $T_{i}$ defines a $K_{2}$-prime in $R_{2}$.

We turn to assertion (ii). As just observed, the degree zero part $\left(\left(R_{2}\right)_{T_{r_{1}+1} \cdots T_{r_{2}}}\right)_{0}$ of the $K_{2}^{\prime}$-grading is isomorphic to $R_{1}$ and thus integral (normal if $R_{1}$ is). Moreover, the $K_{2}^{\prime}$-grading is free in the sense that the associated torus Spec $\mathbb{K}\left[K_{2}^{\prime}\right]$ acts freely on $\operatorname{Spec}\left(R_{2}\right)_{T_{r_{1}+1} \cdots T_{r_{2}}}$. It follows that $\left(R_{2}\right)_{T_{r_{1}+1} \cdots T_{r_{2}}}$ is integral (normal if $R_{1}$ is). Construction 3.1 gives that $R_{2}$ is integral. Moreover, if $T_{r_{1}+1}, \ldots, T_{r_{2}}$ define primes in $R_{2}$, then we can conclude that $R_{2}$ is normal.

Let us apply the results to Cox rings. We first briefly recall the basic definitions and facts; for details, we refer to [1]. For a normal variety $X$ with finitely generated divisor class group $\mathrm{Cl}(X)$ and $\Gamma\left(X, \mathcal{O}^{*}\right)=\mathbb{K}^{*}$, its Cox ring is defined as the graded ring

$$
\mathcal{R}(X):=\bigoplus_{\mathrm{Cl}(X)} \Gamma(X, \mathcal{O}(D)) .
$$

This ring is normal and factorially $\mathrm{Cl}(X)$-graded. Moreover, if $\mathcal{R}(X)$ is finitely generated, then we can reconstruct $X$ from $\mathcal{R}(X)$ as a good quotient of an open subset of $\operatorname{Spec} \mathcal{R}(X)$ by the action of $\operatorname{Spec} \mathbb{K}[\mathrm{Cl}(X)]$.

Now return to the setting fixed at the beginning of the section and assume in addition that the columns of $P_{2}$ are pairwise different primitive vectors in $\mathbb{Z}^{n}$ and those of $P_{1}$ generate $\mathbb{Q}^{n}$ as a convex cone. Suppose that we have toric Cox constructions $\pi_{i}: \hat{Z}_{i} \rightarrow Z_{i}$, where $\hat{Z}_{i} \subseteq \mathbb{K}^{r_{i}}$ are open toric subvarieties, and $\pi_{i}$ are toric morphisms defined by $P_{i}$; see [7]. Then the canonical map $Z_{2} \rightarrow Z_{1}$ is a toric modification. Consider the ideal $I_{1}$ as discussed before and the geometric data

$$
\bar{X}_{1}:=V\left(I_{1}\right) \subseteq \mathbb{K}^{r_{1}}, \quad \hat{X}_{1}:=\bar{X}_{1} \cap \hat{Z}_{1}, \quad X_{1}:=\pi_{1}\left(\hat{X}_{1}\right) \subseteq Z_{1} .
$$

Assume that $R_{1}$ is normal and factorially $K_{1}$-graded and $T_{1}, \ldots, T_{r_{1}}$ define pairwise nonassociated prime elements in $R_{1}$. Then $R_{1}$ is the Cox ring of $X_{1}$ by [1, Thm. 3.2.1.4]. Our statement concerns the Cox ring of the proper transform $X_{2} \subseteq Z_{2}$ of $X_{1} \subseteq Z_{1}$ with respect to $Z_{2} \rightarrow Z_{1}$.

COROllary 3.4. In this setting, assume that $R_{2}$ is normal and the variables $T_{1}, \ldots, T_{r_{2}}$ define pairwise nonassociated $K_{2}$-prime elements in $R_{2}$. Then the $K_{2}$ graded ring $R_{2}$ is the Cox ring of $X_{2}$.

Proof. According to Theorem 3.2, the ring $R_{2}$ is factorially $K_{2}$-graded. Moreover, with the toric Cox construction $\pi_{2}: \hat{Z}_{2} \rightarrow Z_{2}$, we obtain that $R_{2}$ is the algebra of functions of the closure $\hat{X}_{2} \subseteq \hat{Z}_{2}$ of $\pi_{2}^{-1}\left(X_{2} \cap \mathbb{T}^{r_{2}}\right)$. Thus, [1, Thm. 3.2.1.4] yields that $R_{2}$ is the Cox ring of $X_{2}$.

EXAMPLE 3.5. We start with the UFD $R_{1}=\mathbb{K}\left[T_{1}, \ldots, T_{8}\right] / I_{1}$, where the ideal $I_{1}$ is defined as

$$
I_{1}=\left\langle T_{1} T_{2}+T_{3} T_{4}+T_{5} T_{6}+T_{7} T_{8}\right\rangle
$$


The ideal $I_{1}$ is homogeneous with respect to the standard grading given by $Q_{1}=$ $[1, \ldots, 1]$. A Gale dual is $P_{1}=\left[e_{0}, e_{1}, \ldots, e_{7}\right]$, where $e_{0}=-e_{1}-\cdots-e_{7}$ and $e_{i}$ are the canonical basis vectors. Concatenating $e_{1}+e_{3}$ gives a matrix $P_{2}$. The resulting UFD is $R_{2}=\mathbb{K}\left[T_{1}, \ldots, T_{9}\right] / I_{2}$ with

$$
I_{2}=\left\langle T_{1} T_{2} T_{9}+T_{3} T_{4} T_{9}+T_{5} T_{6}+T_{7} T_{8}\right\rangle
$$

\section{Proof of Theorem 1.2}

We approach the Chow quotient via toric embedding. The idea then is to obtain the Cox ring via toric ambient modifications. An essential step for this is an explicit description of the rays of certain Gelfand-Kapranov-Zelevinsky decompositions given in Proposition 4.1; note that in the setting of polytopes, related statements implicitly occur in literature (e.g., [11; 12]).

Recall that the Gelfand-Kapranov-Zelevinsky decomposition associated with a matrix $P \in \operatorname{Mat}(n, r+1 ; \mathbb{Z})$ is the fan $\Sigma$ in $\mathbb{Q}^{n}$ with the cones $\sigma(v)=\bigcap_{v \in \tau^{\circ}} \tau$, where $v \in \mathbb{Q}^{n}$, and $\tau$ runs through the $P$-cones, that is, the cones generated by some of the columns $p_{0}, \ldots, p_{r}$ of $P$. Fix a Gale dual matrix $Q \in \operatorname{Mat}(k, r+$ $1 ; \mathbb{Z})$, where $r+1=k+n$, and denote the columns of $Q$ by $q_{0}, \ldots, q_{r}$. Then we have mutually dual exact sequences of rational vector spaces

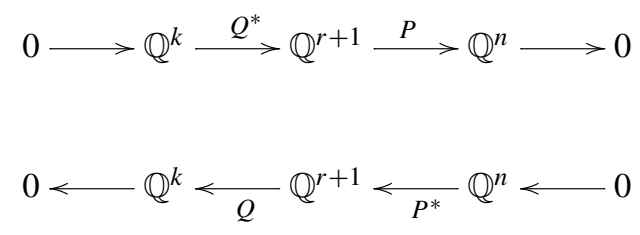

By a $Q$-hyperplane we mean a linear hyperplane in $\mathbb{Q}^{k}$ generated by some of the columns $q_{0}, \ldots, q_{r}$. Given a $Q$-hyperplane, we write it as the kernel $u^{\perp}$ of a linear form $u$ and associate with it a ray in $\mathbb{Q}^{n}$ as follows:

$$
\varrho(u):=\text { cone }\left(\sum_{u\left(q_{i}\right)>0} u\left(q_{i}\right) p_{i}\right) .
$$

It turns out that $\varrho(u)=\varrho(-u)$ and thus the ray is well defined. We say that a column $q_{i}$ of $Q$ is extremal if it does not belong to the relative interior of the "movable cone" $\bigcap_{i} \operatorname{cone}\left(q_{j} ; j \neq i\right)$.

Proposition 4.1. Let $Q$ and $P$ be Gale dual matrices as before, assume that the columns of $P$ are pairwise linearly independent nonzero vectors generating $\mathbb{Q}^{n}$ as a cone, and let $\Sigma$ be the Gelfand-Kapranov-Zelevinsky decomposition associated with $P$.

(i) If a ray $\varrho \in \Sigma$ is the intersection of two $P$-cones, then $\varrho=\varrho(u)$ with a $Q$ hyperplane $u^{\perp}$.

(ii) If $k=2$, then every ray of $\Sigma$ can be obtained as an intersection of two $P$ cones. 
(iii) Assume that $k=2$ and fix nonzero linear forms $u_{i}$ with $u_{i} \perp q_{i}$. Then the rays of $\Sigma$ are cone $\left(p_{0}\right), \ldots$, cone $\left(p_{r}\right)$ and the $\varrho\left(u_{i}\right)$ with $q_{i}$ not extremal.

The proof relies on the fact that $\Sigma$ describes the lifts of regular $Q$-subdivisions. We adapt the precise formulation of this statement to our needs. Let $\gamma \subseteq \mathbb{Q}^{r+1}$ be the positive orthant and define a $\gamma$-collection to be a set $\mathfrak{B}$ of faces of $\gamma$ such that any two $\gamma_{1}, \gamma_{2} \in \mathfrak{B}$ admit an invariant separating linear form $f$ in the sense that

$$
P^{*}\left(\mathbb{Q}^{n}\right) \subseteq f^{\perp}, \quad f_{\mid \gamma_{1}} \geq 0, \quad f_{\mid \gamma_{2}} \leq 0, \quad f^{\perp} \cap \gamma_{i}=\gamma_{1} \cap \gamma_{2} .
$$

Write $\mathfrak{B}_{1} \leq \mathfrak{B}_{2}$ if for every $\gamma_{1} \in \mathfrak{B}_{1}$, there is a $\gamma_{2} \in \mathfrak{B}_{2}$ with $\gamma_{1} \subseteq \gamma_{2}$. Moreover, call a $\gamma$-collection $\mathfrak{B}$ normal if it cannot be enlarged as a $\gamma$-collection and the images $Q\left(\gamma_{0}\right)$, where $\gamma_{0} \in \mathfrak{B}$, form the normal fan of a polyhedron. For a face $\gamma_{0} \preceq \gamma$, we denote by $\gamma_{0}^{*}=\gamma_{0}^{\perp} \cap \gamma^{\vee}$ the corresponding face of the dual cone $\gamma^{\vee}$.

Now assume that the columns of $P$ are pairwise different nonzero vectors. Then [1, Sect. 2.2] provides us with an order-reversing bijection

$$
\{\text { normal } \gamma \text {-collections }\} \rightarrow \Sigma, \quad \mathfrak{B} \mapsto \bigcap_{\gamma_{0} \in \mathfrak{B}} P\left(\gamma_{0}^{*}\right) \text {. }
$$

Proof of Proposition 4.1. We prove (i). Let $\varrho=P\left(\gamma_{1}^{*}\right) \cap P\left(\gamma_{2}^{*}\right)$ with $\gamma_{1}, \gamma_{2} \preceq \gamma$. We may assume that the relative interiors $P\left(\gamma_{1}^{*}\right)^{\circ}$ and $P\left(\gamma_{2}^{*}\right)^{\circ}$ intersect nontrivially. Then $\gamma_{1}$ and $\gamma_{2}$ admit an invariant separating linear form $f=Q^{*}(u)$ with a linear form $u$ on $\mathbb{Q}^{k}$. In terms of the components of $f_{i}=u\left(q_{i}\right)$ of $f$, we have

$$
\gamma_{1}=\text { cone }\left(e_{i} ; f_{i} \geq 0\right), \quad \gamma_{2}=\operatorname{cone}\left(e_{i} ; f_{i} \leq 0\right) \text {. }
$$

Write $f=f^{+}-f^{-}$with the unique vectors $f^{+}, f^{-} \in \mathbb{Q}^{r+1}$ having only nonnegative components. Then $P(f)=0$ gives $P\left(f^{+}\right)=P\left(f^{-}\right)$. We conclude that $\varrho=\operatorname{cone}\left(P\left(f^{+}\right)\right)$, and the assertion follows.

We prove (ii) and (iii). The rays of $\Sigma$ arise from normal $\gamma$-collections that are submaximal with respect to " $\leq$ " in the sense that the only dominating $\gamma$-collection is the trivial collection $\langle\gamma\rangle$ consisting of all faces $\gamma_{0} \preceq \gamma$ that are invariantly separable from $\gamma$. There are precisely two types of such submaximal collections:

- the normal $\gamma$-collections $\mathfrak{B}=\left\langle\gamma_{0}\right\rangle$, where $\gamma_{0} \supsetneqq \gamma$ is a facet satisfying $Q\left(\gamma_{0}\right)=$ $Q(\gamma)$

- the normal $\gamma$-collections $\mathfrak{B}=\left\langle\gamma_{1}, \gamma_{2}\right\rangle$, where $\gamma_{1}, \gamma_{2} \supsetneqq \gamma$ are invariantly separable from each other and satisfy

$$
\gamma_{i}=Q^{-1}\left(Q\left(\gamma_{i}\right)\right) \cap \gamma, \quad Q(\gamma)=Q\left(\gamma_{1}\right) \cup Q\left(\gamma_{2}\right) .
$$

The submaximal $\gamma$-collections of the first type give the rays cone $\left(p_{i}\right) \in \Sigma$ with $q_{i}$ not extremal. If $q_{i}$ is extremal, then the (unique) $\gamma$-collection of the second type with $Q\left(\gamma_{1}\right)=\operatorname{cone}\left(q_{j} ; j \neq i\right)$ defines the ray cone $\left(p_{i}\right)$. The remaining rays of $\Sigma$ are of the form $\varrho=P\left(\gamma_{1}^{*}\right) \cap P\left(\gamma_{2}\right)^{*}$ with the remaining collections of the second type.

REMARK 4.2. Statements (ii) and (iii) of Proposition 4.1 hold as well for pairs $P$, $Q$, where the columns of $Q$ generate the cone over a so-called totally-2-splittable polytope; these have been studied in $[11 ; 12]$. 
As a further preparation of the proof of Theorem 1.2, we have to specialize the discussion of Section 3 to the case of a single defining equation. The following notion will be used for an explicit description of the transferred ideal.

Definition 4.3. Consider an $n \times(r+1)$ matrix $P$ and an $n \times l$ matrix $B$, both integral. A weak $B$-lifting (with respect to $P$ ) is an integral $(r+1) \times l$ matrix $A$ allowing a commutative diagram

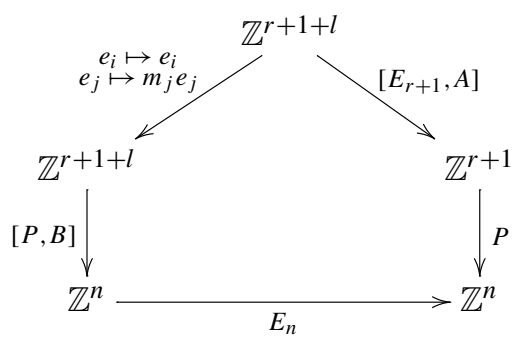

where $e_{i}$ are the first $r+1$ and $e_{j}$ the last $l$ canonical basis vectors of $\mathbb{Z}^{r+1+l}$, $m_{j}$ are positive integers, and $E_{n}$ and $E_{r+1}$ denote the unit matrices of sizes $n$ and $r+1$, respectively.

Note that weak $B$-liftings $A$ always exist. Given such $A$, consider the following homomorphism of Laurent polynomial rings:

$$
\begin{aligned}
\psi_{A}: \mathbb{K}\left[T_{0}^{ \pm 1}, \ldots, T_{r}^{ \pm 1}\right] & \rightarrow \mathbb{K}\left[T_{0}^{ \pm 1}, \ldots, T_{r}^{ \pm 1}, S_{1}^{ \pm 1}, \ldots, S_{l}^{ \pm 1}\right], \\
\sum \alpha_{v} T^{v} & \mapsto \sum \alpha_{v} T^{v} S^{A^{t} \cdot v} .
\end{aligned}
$$

Set $K_{1}:=\mathbb{Z}^{r+1} / P^{*}\left(\mathbb{Z}^{n}\right)$. Then the left-hand side algebra is $K_{1}$-graded by assigning to the $i$ th variable the class of $e_{i}$ in $K_{1}$.

Lemma 4.4. In the previous notation, let $g_{1} \in \mathbb{K}\left[T_{0}^{ \pm 1}, \ldots, T_{r}^{ \pm 1}\right]$ be a $K_{1}$ homogeneous polynomial.

(i) We have $T^{v} S^{\mu} \psi_{A}\left(g_{1}\right)=g_{2}^{\prime}$ with $v \in \mathbb{Z}^{r+1}, \mu \in \mathbb{Z}^{l}$ and a unique monomialfree $g_{2}^{\prime} \in \mathbb{K}\left[T_{0}, \ldots, T_{r}, S_{1}, \ldots, S_{l}\right]$.

(ii) The polynomial $g_{2}^{\prime}$ is of the form $g_{2}^{\prime}=g_{2}\left(T_{0}, \ldots, T_{r+1}, S_{1}^{m_{1}}, \ldots, S_{l}^{m_{1}}\right)$ with a $g_{2} \in \mathbb{K}\left[T_{0}, \ldots, T_{r}, S_{1}, \ldots, S_{l}\right]$ not depending on the choice of $A$.

(iii) If, in the setting of Construction 3.1, we have $I_{1}=\left\langle g_{1}\right\rangle$, then the transferred ideal is given by $I_{2}=\left\langle g_{2}\right\rangle$.

(iv) The variable $T_{i}$ defines a prime element in $\mathbb{K}\left[T_{0}, \ldots, T_{r+l+1}\right] /\left\langle g_{2}\right\rangle$ if and only if the polynomial $g_{2}\left(T_{1}, \ldots, T_{i-1}, 0, T_{i+1}, \ldots, T_{r+l+1}\right)$ is irreducible.

Proof. Consider the commutative diagram of group algebras corresponding to the dualized diagram (Definition 4.3). There, $\psi_{A}$ occurs as the homomorphism of group algebras defined by the transpose $\left[E_{r+1}, A\right]^{*}$. Let $T^{\kappa}$ be any monomial of $g_{1}$. Then $g_{1}^{\prime}:=T^{-\kappa} g_{1}$ gives rise to the same $g_{2}$, but $g_{1}^{\prime}$ is of $K_{1}$-degree zero and hence a pullback $g_{1}^{\prime}=\psi_{P^{*}}(h)$. The latter allows us to use commutativity of the diagram, which gives (i) and (ii). Assertions (iii) and (iv) are clear. 
Proof of Theorem 1.2. Recall that we consider the quadric $X=V\left(g_{1}\right) \subseteq \mathbb{P}_{r}$ with $g_{1}=T_{0} T_{1}+\cdots+T_{r-1} T_{r}$, where we replace the last term with $T_{r}^{2}$ in the case of an even $r$, and a $\mathbb{K}^{*}$-action on $\mathbb{P}_{r}$, given by weights $\zeta_{0}, \ldots, \zeta_{r}$ such that $g_{0}$ is of degree zero and, in particular, $X$ is invariant.

In a first step, we construct a suitable GIT quotient $X_{1}$ of the $\mathbb{K}^{*}$-action on $X$. Lifting the data to $\mathbb{K}^{r+1}$ gives $\bar{X}:=V\left(g_{1}\right) \subseteq \mathbb{K}^{r+1}$, which is invariant under the action of $\mathbb{T}^{2}=\mathbb{K}^{*} \times \mathbb{K}^{*}$ on $\mathbb{K}^{r+1}$ given by the weight matrix

$$
Q:=\left[\begin{array}{ccc}
\zeta_{0} & \ldots & \zeta_{r} \\
1 & \ldots & 1
\end{array}\right]
$$

Consider the weight $w=(0,1)$ of $\mathbb{T}^{2}$ and the associated set of semistable points $\hat{Z}_{1} \subseteq \mathbb{K}^{r+1}$, that is, the union of all localizations $\mathbb{K}_{f}^{r+1}$, where $f$ is homogeneous with respect to some positive multiple of $w$. Then $\hat{Z}_{1}$ is a toric open subset, and with $\hat{X}_{1}:=\bar{X} \cap \hat{Z}_{1}$, we obtain a commutative diagram

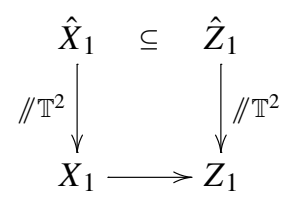

where the induced map $X_{1} \rightarrow Z_{1}$ of quotients is a closed embedding. We are in the setting presented before Corollary 3.4. In particular, $\hat{Z}_{1} \rightarrow Z_{1}$ is a toric Cox construction with a Gale dual $P$ of $Q$ as describing matrix; note that the columns of $P$ generate $\mathbb{Z}^{r-1}$ as a lattice. Moreover, the Cox ring of $X_{1}$ is the $\mathbb{Z}^{2}$-graded ring

$$
R_{1}=\mathbb{K}\left[T_{0}, \ldots, T_{r}\right] /\left\langle g_{1}\right\rangle .
$$

Observe that $X_{1}$ is as well the $\mathbb{K}^{*}$-quotient of the image of $\hat{X}_{1}$ in $X$, which in turn is the set of semistable points of a suitable linearization of $\mathcal{O}(1)$.

Set $n:=r-1$ and consider the Gelfand-Kapranov-Zelevinsky decomposition $\Sigma$ associated to $P$. Then, according to Proposition 2.4 , the toric variety $Z_{2}$ determined by $\Sigma$ is the normalized Chow quotient of the $\mathbb{K}^{*}$-action on $\mathbb{P}_{r}$. Moreover, let $X_{2} \subseteq Z_{2}$ denote the proper transform of $X_{1} \subseteq Z_{1}$ under the toric morphism $Z_{2} \rightarrow Z_{1}$. Then Proposition 2.5 tells us that $X_{2}$ and the Chow quotient $X_{\text {ce }}^{\prime} \mathbb{K}^{*}$ share the same normalization.

We will now show that $X_{2}$ is in fact normal and that its Cox ring is as claimed in the theorem. As before, put the primitive generators $b_{1}, \ldots, b_{l}$ of rays of $\Sigma$ differing from columns of $P$ into a matrix $B$ and choose a weak $B$-lifting $A$ with respect to $P$; using the fact that the columns of $P$ generate $\mathbb{Z}^{n}$, we can choose the numbers $m_{j}$ all equal to one. With the shifted row sums $\eta_{0}, \eta_{2}, \ldots, \eta_{r-1}$, we set

$$
g_{2}:= \begin{cases}T_{0} T_{1} S^{\eta_{0}}+T_{2} T_{3} S^{\eta_{2}}+\cdots+T_{r-1} T_{r} S^{\eta_{r-1}}, & r \text { odd, } \\ T_{0} T_{1} S^{\eta_{0}}+\cdots+T_{r-2} T_{r-1} S^{\eta_{r-2}}+T_{r}^{2} S^{\eta_{r}}, & r \text { even. }\end{cases}
$$

Lemma 4.4 then ensures that $I_{2}:=\left\langle g_{2}\right\rangle$ is the transferred ideal of $I_{1}:=\left\langle g_{1}\right\rangle$ in the sense of Construction 3.1; define $P_{1}:=P$ and $P_{2}:=[P, B]$ to adapt the settings. 
Consider the ring

$$
R_{2}=\mathbb{K}\left[T_{0}, \ldots, T_{r}, S_{1}, \ldots, S_{l}\right] /\left\langle g_{2}\right\rangle
$$

Our task is to show that the variables $S_{1}, \ldots, S_{l}$ define prime elements in $R_{2}$. Then Proposition 3.3 tells us that $R_{2}$ and thus $X_{2}$ are normal, and Corollary 3.4 yields that the Cox ring of $X_{2}$ is $R_{2}$ together with the $\mathbb{Z}^{2+l}$-grading defined by a Gale dual $Q_{2}$ of $P_{2}=[P, B]$.

Suitably renumbering the variables $T_{i}$, we achieve that $\left|\zeta_{r-3}\right|, \ldots,\left|\zeta_{r}\right|$ are minimal among all $\left|\zeta_{i}\right|$ in the case of odd $r$ and, similarly, in the case of even $r$, we have $\zeta_{r-3}=\zeta_{r-2}=\zeta_{r-1}=0$. In order to see that the $S_{j}$ define primes, it suffices to show that, according to odd and even $r$,

$$
g_{2}=T_{r-3} T_{r-2}+T_{r-1} T_{r}+h, \quad \text { or } \quad g_{2}=T_{r-2} T_{r-1}+T_{r}^{2}+h
$$

with a polynomial $h \in \mathbb{K}\left[T_{0}, \ldots, T_{r}, S_{1}, \ldots, S_{l}\right]$ not depending on the last four (three) $T_{i}$; see Lemma 4.4(iv). This in turn is seen by constructing a suitable weak $B$-lifting via the description of the rays through $b_{1}, \ldots, b_{l}$ provided by Proposition 4.1. Each $b_{j}$ (or a suitable integral multiple) stems from a $Q$-hyperplane, and the $u_{j}$ can be chosen to be nonpositive on the last four (three) $q_{i}$. Putting $\max \left(0, u_{j}\left(q_{i}\right)\right)$ into a matrix $A^{\prime}$ gives a weak $B$-lifting $A^{\prime}$ with $A_{i *}^{\prime}=0$ for the last four (three) rows. By Lemma 4.4 the weak $B$-lifting $A^{\prime}$ yields the same $g_{2}$, which now has the desired form.

EXAMPLE 4.5. Consider the quadric $X=V\left(T_{0} T_{1}+T_{2} T_{3}+T_{4} T_{5}+T_{6}^{2}\right) \subseteq \mathbb{P}_{6}$ and the action of $\mathbb{K}^{*}$ on $\mathbb{P}_{6}$ given by

$$
t \cdot\left[x_{0}, \ldots, x_{6}\right]:=\left[t^{-2} x_{0}, t^{2} x_{1}, t^{-1} x_{2}, t^{1} x_{3}, x_{4}, x_{5}, x_{6}\right] .
$$

An integral Gale dual $P$ of the extended weight matrix $Q$ is of size $5 \times 7$ and explicitly given as

$$
\left[\begin{array}{ccccccc}
-1 & -1 & 1 & 1 & 0 & 0 & 0 \\
0 & 0 & 0 & 0 & -1 & 1 & 0 \\
0 & -1 & -1 & 1 & 0 & 1 & 0 \\
0 & 0 & 1 & 1 & -1 & -1 & 0 \\
0 & 0 & 0 & 0 & -1 & 0 & 1
\end{array}\right]
$$

Computing the associated Gelfand-Kapranov-Zelevinsky decomposition, we see that it comes with one new ray, namely

$$
b_{1}=(-1,0,-1,1,0)=2 p_{0}+p_{2},
$$

where $p_{0}, \ldots, p_{6}$ are the columns of $P$. The Cox ring of the normalized Chow quotient $X_{\mathrm{cQ}}^{\tilde{l}} \mathbb{K}^{*}$ is the ring

$$
\mathcal{R}\left(X_{\mathrm{cQ}}^{\tilde{c}} \mathbb{K}^{*}\right)=\mathbb{K}\left[T_{0}, \ldots, T_{6}, S_{1}\right] /\left\langle T_{0} T_{1} S_{1}^{2}+T_{2} T_{3} S_{1}+T_{4} T_{5}+T_{6}^{2}\right\rangle
$$


together with the grading by $\mathrm{Cl}(X)=\mathbb{Z}^{3}$ via a Gale dual of $\left[p_{0}, \ldots, p_{6}, b_{1}\right]$, that is, the degrees of the variable are the columns of

$$
\left[\begin{array}{cccccccc}
-2 & 2 & -1 & 1 & 0 & 0 & 0 & 0 \\
1 & 1 & 1 & 1 & 1 & 1 & 1 & 0 \\
2 & 0 & 1 & 0 & 0 & 0 & 0 & -1
\end{array}\right]
$$

REMARK 4.6. The setting of Theorem 1.2 can also be interpreted in terms of Mori theory, especially in the sense of [14]. There are (up to isomorphism) finitely many normal projective varieties $Y_{1}, \ldots, Y_{s}$ sharing as their Cox ring a given $R_{1}=\mathbb{K}\left[T_{0}, \ldots, T_{n}\right] /\left\langle g_{1}\right\rangle$ with its $\mathbb{Z}^{2}$-grading coming from the extended weight matrix $Q$. Each $Y_{i}$ is a GIT-quotient of the induced $\mathbb{K}^{*}$-action on the quadric $X=$ $V\left(g_{1}\right) \subseteq \mathbb{P}_{r}$ and thus dominated in universal manner by the normalized Chow quotient $Y=X_{\text {cQ }}^{\tilde{I}} \mathbb{K}^{*}$. Thus, $Y$ is the "Mori master space" controlling the whole class of small birational relatives $Y_{i}$. This picture obviously extends to all Mori dream spaces, and it is a natural desire to study the geometry of such Mori master spaces.

REMARK 4.7. The assumption in Theorem 1.2 that, for odd (even) $r$, there are at least four (three) weights $\zeta_{i}$ of minimal absolute value is used for verifying the primality conditions on the variables $T_{i}$ in Corollary 3.4. It would be interesting to see what happens beyond this assumption. We expect that the Cox ring then has further generators, in addition to the variables $S_{j}$; note that in the setting of Remark 4.6, the $S_{j}$ correspond to the canonical sections of exceptional divisors of $Y \rightarrow Y_{i}$ for some fixed $i$.

\section{Proof of Theorem 1.1}

The main idea of the proof is to consider, instead of the Chow quotient, its "weak tropical resolution" and to use intrinsic symmetry of the latter space. This approach applies also to problems beyond $\mathbb{K}^{*}$-actions on quadrics; we therefore develop it in sufficient generality. We begin with recalling the necessary concepts from tropical geometry.

Let $f$ be a Laurent polynomial in $n$ variables. The Newton polytope $B_{f} \subseteq \mathbb{Q}^{n}$ is the convex hull over the exponent vectors of $f$. The tropical variety $\operatorname{trop}(V(f))$ of the zero set $V(f) \subseteq \mathbb{T}^{n}$ lives in $\mathbb{Q}^{n}$ and is defined to be the union of all $(n-1)$ dimensional cones of the normal fan of $B_{f}$. The tropical variety of an arbitrary closed subset $Y \subseteq \mathbb{T}_{n}$ is the intersection trop $(Y)$ over all trop $(V(f))$, where $f$ runs through the ideal of $Y$. It turns out that $\operatorname{trop}(Y)$ is the support of an (in general not unique and not pointed) fan in $\mathbb{Q}^{n}$.

Definition 5.1. Consider a toric variety $Z$ defined by a fan $\Sigma$ in $\mathbb{Q}^{n}$ and an irreducible subvariety $Y \subseteq Z$ intersecting the big torus $\mathbb{T}^{n} \subseteq Z$ nontrivially. We call the embedding $Y \subseteq Z$ weakly tropical if the support $|\Sigma| \subseteq \mathbb{Q}^{n}$ equals the tropical variety $\operatorname{trop}\left(Y \cap \mathbb{T}^{n}\right) \subseteq \mathbb{Q}^{n}$. 
Remark 5.2. Any tropical embedding in the sense of Tevelev [22] is weakly tropical. If $Y \subseteq Z$ is a weakly tropical subvariety of a toric variety $Z$, then, by [10, Sect. 14], for any toric orbit $\mathbb{T}^{n} \cdot z \subseteq Z$ intersecting $Y$ nontrivially, we have

$$
\operatorname{dim}(Z)-\operatorname{dim}\left(\mathbb{T}^{n} \cdot z\right)=\operatorname{dim}(Y)-\operatorname{dim}\left(\mathbb{T}^{n} \cdot z \cap Y\right) .
$$

Construction 5.3 (Weak tropical resolution). Let $Z$ be a complete toric variety arising from a fan $\Sigma$ in $\mathbb{Q}^{n}$, and $Y \subseteq Z$ an irreducible subvariety intersecting the big torus $\mathbb{T}^{n} \subseteq Z$ nontrivially. Fix a fan structure $\Sigma_{Y}$ carried on the tropical variety $\operatorname{trop}\left(Y \cap \mathbb{T}^{n}\right) \subseteq \mathbb{Q}^{n}$ for $Y \cap \mathbb{T}^{n}$ and consider the coarsest common refinement

$$
\Sigma^{\prime}:=\Sigma \sqcap \Sigma_{Y}=\left\{\tau \cap \sigma ; \sigma \in \Sigma, \tau \in \Sigma_{Y}\right\}
$$

of the fans $\Sigma$ and $\Sigma_{Y}$. Then the canonical map of fans $\Sigma^{\prime} \rightarrow \Sigma$ defines a birational toric morphism $Z^{\prime} \rightarrow Z$ of the associated toric varieties. With the proper transform $Y^{\prime} \subseteq Z^{\prime}$ of $Y \subseteq Z$, we obtain a proper birational map $Y^{\prime} \rightarrow Y$, which we call a weak tropical resolution of $Y \subseteq Z$.

Proof. The only thing to show is the properness of the morphism $Y^{\prime} \rightarrow Y$. But this follows directly from Tevelev's criterion [22, Prop. 2.3].

The use of passing to the weak tropical resolution in our context is that it enables us to divide out torus symmetries in a controlled manner. This leads to an explicit version of [18, Thm. 1.2] relating the Mori dream space property of a variety to the Mori dream space property of a certain quotient.

Construction 5.4. Consider a toric variety $Z$ arising from a fan $\Sigma$ in $\mathbb{Q}^{r}$ and a weakly tropical embedded subvariety $Y \subseteq Z$. Suppose that $Y$ is invariant under the action of a subtorus $T \subseteq \mathbb{T}^{r}$. Set

$$
Z_{0}:=\left\{z \in Z ; \operatorname{dim}\left(\mathbb{T}^{r} \cdot z\right) \geq r-1, T_{z} \text { finite }\right\}, \quad Y_{0}:=Y \cap Z_{0} .
$$

Then $Z_{0} \subseteq Z$ is an open toric subset corresponding to a subfan $\Sigma_{0} \preceq \Sigma$ with certain rays $\varrho_{1}, \ldots, \varrho_{s}$ of $\Sigma$ as its maximal cones. Let the matrix $P \in \operatorname{Mat}(n, r ; \mathbb{Z})$ describe an epimorphism $\pi: \mathbb{T}^{r} \rightarrow \mathbb{T}^{n}$ with $\operatorname{ker}(\pi)=T$ and consider the following fan in $\mathbb{Z}^{n}$ :

$$
\Delta_{0}:=\left\{0, P\left(\varrho_{1}\right), \ldots, P\left(\varrho_{s}\right)\right\} .
$$

Note that $\varrho_{1}, \ldots, \varrho_{s}$ are precisely the rays of $\Sigma$ that are not contained in $\operatorname{ker}(P)$. The matrix $P$ determines a toric morphism $Z_{0} \rightarrow Z_{0}^{\prime} T$ onto the toric variety associated to $\Delta_{0}$. We define $Y_{0}^{\prime} T \subseteq Z_{0}^{\prime} T$ to be the closure of the image $\pi\left(Y \cap \mathbb{T}^{r}\right)$.

Remark 5.5. The tropical variety trop $\left(Y_{0}^{\prime} T \cap \mathbb{T}^{n}\right)$ contains all rays $P\left(\varrho_{1}\right), \ldots$, $P\left(\varrho_{s}\right)$ of the fan $\Delta_{0}$. If there is a fan $\Delta$ in $\mathbb{Z}^{n}$ having $\operatorname{trop}\left(Y_{0}^{\prime} T \cap \mathbb{T}^{n}\right)$ as its support and $P\left(\varrho_{1}\right), \ldots, P\left(\varrho_{s}\right)$ as its rays, then $Y_{0}^{\prime} T$ admits a weakly tropical completion with boundary of codimension at least two. 
Proposition 5.6. Consider a toric variety $Z$ and a weakly tropical subvariety $Y \subseteq Z$. Suppose that $Y$ is invariant under the action of a subtorus $T \subseteq \mathbb{T}^{r}$. Then the following statements are equivalent:

(i) The normalization of $Y$ has finitely generated Cox ring.

(ii) The normalization of $Y_{0}^{\prime} T$ has finitely generated Cox ring.

Proof. Let $v: \tilde{Y} \rightarrow Y$ be the normalization map. By $W \subseteq Y$ we denote the open $T$-invariant subset consisting of all points $y \in Y$ having a finite isotropy group $T_{y}$. The fact that $Y \subseteq Z$ is tropically embedded ensures that $Y_{0} \subseteq W$ has a complement of codimension at least two in $W$. This property is preserved when passing to the respective normalizations $\tilde{W}:=v^{-1}(W)$ and $\tilde{Y}_{0}:=v^{-1}\left(Y_{0}\right)$. In particular, the separations in the sense of $[18$, p. 978$]$ of the corresponding quotients $\tilde{W} / T$ and $\tilde{Y}_{0} / T$ have the same Cox rings. Since normalizing commutes with taking quotients and separating, the latter space is isomorphic to the normalization of $Y_{0}^{\prime} T$. Thus, the assertion follows from [18, Thm. 1.2].

Proposition 5.7. Let $Z$ be a toric variety, $Y \subseteq Z$ a complete subvariety invariant under a subtorus $T$ of the big torus of $Z$, and $Y^{\prime} \rightarrow Y$ be a weak tropical resolution. If the normalization of $Y_{0}^{\prime}, T$ has finitely generated Cox ring, then the normalization $\tilde{Y}$ of $Y$ is a Mori dream space.

Proof. Since the normalization of $Y_{0}^{\prime} T$ has finitely generated Cox ring, Proposition 5.6 shows that the normalization $Y^{\prime \prime}$ of $Y^{\prime}$ has finitely generated Cox ring and thus is a Mori dream space. The canonical morphism $\pi: Y^{\prime \prime} \rightarrow \tilde{Y}$ is proper and birational. In order to see that $\tilde{Y}$ is a Mori dream space, we may apply the general [20, Thm. 10.4] or look at a suitable sheaf $\mathcal{S}=\bigoplus_{K} \mathcal{O}_{\tilde{Y}}(D)$ of divisorial algebras on $\tilde{Y}$ mapping onto the Cox sheaf $\mathcal{R}$ of $\tilde{Y}$. By the properness of $\pi$ we obtain $\mathcal{S}=\pi_{*} \mathcal{S}^{\prime \prime}$ over the set $W \subseteq \tilde{Y}$ of regular points for $\mathcal{S}^{\prime \prime}=\bigoplus_{K} \mathcal{O}_{Y^{\prime \prime}}\left(\pi^{*}(D)\right)$. Since $Y^{\prime \prime}$ is a Mori dream space, $\Gamma\left(\pi^{-1}(W), \mathcal{S}^{\prime \prime}\right)$ is finitely generated. This implies finite generation of the Cox $\operatorname{ring} \mathcal{R}(\tilde{Y})=\Gamma(W, \mathcal{R})$.

A second preparation of the proof of Theorem 1.1 concerns toric ambient modification. We will always write $e_{1}, \ldots, e_{n} \in \mathbb{Z}^{n}$ for the canonical basis vectors and set $e_{0}:=-e_{1}-\cdots-e_{n}$. Moreover, we denote by $\Delta(n)$ the fan in $\mathbb{Z}^{n}$ consisting of all cones spanned by at most $n$ of the vectors $e_{0}, \ldots, e_{n}$ and by $\Delta^{\prime}(n) \subseteq \Delta(n)$ the subfan consisting of all cones of dimension at most $n-1$.

Lemma 5.8. Consider nonzero vectors $v_{1}, \ldots, v_{l} \in \mathbb{Q}^{n}$ contained in a maximal cone $\tau \in \Delta(n)$, a cone $\sigma \subseteq \mathbb{Q}^{n}$ generated by some of the vectors $e_{0}, \ldots, e_{n}$, $v_{1}, \ldots, v_{l}$, and a cone $\delta \in \Delta^{\prime}(n)$. Suppose that $\varrho:=\delta \cap \sigma$ is one-dimensional and $\varrho \notin \Delta^{\prime}(n)$. Then $\varrho$ is contained in some facet of $\tau$.

Proof. We may assume that $\tau=\operatorname{cone}\left(e_{1}, \ldots, e_{n}\right)$. Replacing $\delta$ and $\sigma$ with suitable faces, we may assume that $\varrho^{\circ}=\delta^{\circ} \cap \sigma^{\circ}$. The proof uses Gale duality, and we work in the notation of Section 4 . Consider the matrix $P:=$ 
$\left[e_{0}, \ldots, e_{n}, v_{1}, \ldots, v_{l}\right]$ and its Gale dual

$$
Q:=\left[q_{0}, \ldots, q_{n+l}\right]:=\left[\begin{array}{ccccccc}
0 & v_{11} & \cdots & v_{1 n} & -1 & & 0 \\
\vdots & \vdots & & \vdots & & \ddots & \\
0 & v_{l 1} & \cdots & v_{l n} & 0 & & -1 \\
1 & 1 & \cdots & 1 & 0 & \cdots & 0
\end{array}\right] .
$$

Set $r:=n+l$ and let $e_{0}^{\prime}, \ldots, e_{r}^{\prime}$ denote the canonical basis vectors of $\mathbb{Z}^{r+1}$ and $\gamma:=\mathbb{Q}_{\geq 0}^{r+1}$ the positive orthant. Then there are faces $\gamma_{1}, \gamma_{2} \preceq \gamma$ such that for the corresponding dual faces $\gamma_{i}^{*}$, we have

$$
P\left(\gamma_{1}^{*}\right)=\delta, \quad P\left(\gamma_{2}^{*}\right)=\sigma, \quad P\left(\gamma_{1}^{*}\right)^{\circ} \cap P\left(\gamma_{2}^{*}\right)^{\circ} \neq \emptyset .
$$

For some $n+1 \leq j \leq r$, we have $e_{j}^{\prime} \in \gamma_{2}^{*}$, and we may assume that $\gamma_{1}^{*}$ is generated by at most $n-1$ of the vectors $e_{0}^{\prime}, \ldots, e_{n}^{\prime}$. The latter implies $e_{n+1}^{\prime}, \ldots, e_{n+l}^{\prime} \in \gamma_{1}$. Let $f=Q^{*}(u)$ be a separating linear form for $\gamma_{1}$ and $\gamma_{2}$. Then $f_{\mid \gamma_{1}} \geq 0$ implies

$$
u\left(q_{n+1}\right), \ldots, u\left(q_{n+l}\right) \geq 0, \quad u\left(q_{0}\right) \geq u\left(q_{1}\right), \ldots, u\left(q_{n}\right) .
$$

Note that we must have $f\left(e_{j}^{\prime}\right)=u\left(q_{j}\right)>0$ because $e_{j}^{\prime}$ does not lie in $\gamma_{2}$. Let $\tau_{1}, \tau_{2} \preceq \gamma$ be the maximal faces with $f_{\mid \tau_{1}} \geq 0$ and $f_{\mid \tau_{2}} \leq 0$. Then $f$ separates $\tau_{1}$ and $\tau_{2}$, and $\tau_{i}^{*} \subseteq \gamma_{i}^{*}$. We conclude

$$
\emptyset \neq P\left(\tau_{1}^{*}\right)^{\circ} \cap P\left(\tau_{2}^{*}\right)^{\circ} \subseteq P\left(\tau_{1}^{*}\right) \cap P\left(\tau_{2}^{*}\right) \subseteq P\left(\gamma_{1}^{*}\right) \cap P\left(\gamma_{2}^{*}\right)=\varrho .
$$

Since $e_{j}^{\prime} \notin \tau_{2}$, we obtain $\tau_{2}^{*} \neq\{0\}$ and thus $0 \notin P\left(\tau_{2}^{*}\right)^{\circ}$. Together with the displayed line, this gives $P\left(\tau_{1}^{*}\right) \cap P\left(\tau_{2}^{*}\right)=\varrho$. Since at least two of $e_{0}^{\prime}, \ldots, e_{n}^{\prime}$ lie in $\gamma_{1}$, we obtain $e_{0}^{\prime} \in \tau_{1}$, and thus

$$
\varrho \subseteq P\left(\tau_{1}^{*}\right) \subseteq \operatorname{cone}\left(e_{1}, \ldots, e_{n}\right) .
$$

Lemma 5.9. For $n \in \mathbb{Z}_{\geq 1}$, consider $\Delta^{\prime}(n)$ and let $b_{1}, \ldots, b_{l} \in \mathbb{Q}^{n}$ be pairwise different primitive vectors lying on the support of $\Delta^{\prime}(n)$ but not on its rays. Denote by $\sigma_{j} \in \Delta^{\prime}(n)$ the minimal cone with $b_{j} \in \sigma_{j}$ and write

$$
b_{j}=a_{0 j} e_{0}+\cdots+a_{n j} e_{n}, \quad \text { where } a_{i j}>0 \text { if } e_{i} \in \sigma_{j}, a_{i j}=0 \text { if } e_{i} \notin \sigma_{j} .
$$

Then, for $P:=\left[e_{0}, \ldots, e_{n}\right]$ and $B:=\left[b_{1}, \ldots, b_{l}\right]$, the matrix $A:=\left(a_{i j}\right)$ is a weak $B$-lifting with respect to $P$. The lift of $h_{1}=T_{0}+\cdots+T_{n}$ in the sense of Lemma 4.4 is given by

$$
h_{2}=T_{0} S_{1}^{a_{01}} \cdots S_{l}^{a_{0 l}}+\cdots+T_{n} S_{1}^{a_{n 1}} \cdots S_{l}^{a_{n l}} .
$$

Moreover, the variables $T_{0}, \ldots T_{n}, S_{1}, \ldots, S_{l}$ define pairwise nonassociated prime elements in $\mathbb{K}\left[T_{0}, \ldots T_{n}, S_{1}, \ldots, S_{l}\right] /\left\langle h_{2}\right\rangle$ if and only if the vectors $b_{1}, \ldots, b_{l}$ lie in a common cone of $\Delta(n)$.

Proof. Only the last sentence needs some explanation. The fact that $b_{1}, \ldots, b_{l}$ lie in a common cone of $\Delta(n)$ is equivalent to the fact that there is a term of $h_{2}$ not depending on $S_{1}, \ldots, S_{l}$, and, moreover, for every $k$, there is a further term of $h_{2}$ not depending on $S_{k}$. Now, Lemma 4.4(iv) gives the desired characterization. 
Proof of Theorem 1.1. We may assume that $X=V\left(g_{1}\right) \subseteq \mathbb{P}_{r}$ with a polynomial $g_{1}=T_{0} T_{1}+\cdots+T_{r-1} T_{r}$, where we replace the last term with $T_{r}^{2}$ in the case of an even $r$, and $\mathbb{K}^{*}$ acts linearly with weights $\zeta_{0}, \ldots, \zeta_{r}$, where $\left|\zeta_{r}\right|$ is minimal among all $\left|\zeta_{i}\right|$; see [1, Prop. 3.2.4.7].

The first step is to determine the normalized Chow quotient of the $\mathbb{K}^{*}$-action on $X$. As observed in Proposition 2.5, the Chow quotient $X_{\mathrm{c}}^{\prime} \mathbb{K}^{*}$ is canonically embedded into the Chow quotient of $\mathbb{P}_{r}$ by the $\mathbb{K}^{*}$-action. To determine the latter, consider the extended weight matrix

$$
Q:=\left[\begin{array}{rrr}
\zeta_{0} & \ldots & \zeta_{r} \\
1 & \ldots & 1
\end{array}\right]
$$

and let $P$ be a Gale dual matrix. Then, according to Proposition 2.4, the normalized Chow quotient of the $\mathbb{K}^{*}$-action on $\mathbb{P}_{r}$ is the toric variety $Z$ having the Gelfand-Kapranov-Zelevinsky decomposition $\Sigma$ defined by the columns of $P$ as its fan. Moreover, by Proposition 2.5 the Chow quotient of the $\mathbb{K}^{*}$-action on $X$ has the same normalization as the closure

$$
Y=\overline{\left(X \cap \mathbb{T}^{r}\right) / \mathbb{K}^{*}} \subseteq Z
$$

The second step is to determine a weak tropical resolution of $Y \subseteq Z$. For this, we first need $\operatorname{trop}\left(Y \cap T_{Z}\right)$. Let $\mu_{0}, \ldots, \mu_{n} \in \mathbb{Z}^{r+1}$ be the vertices of the Newton polytope $g_{1}$ and consider the matrix $P_{\mathrm{gr}}$ with the rows $\mu_{i}-\mu_{0}, i=1, \ldots, n$. Then we obtain a commutative diagram with exact rows

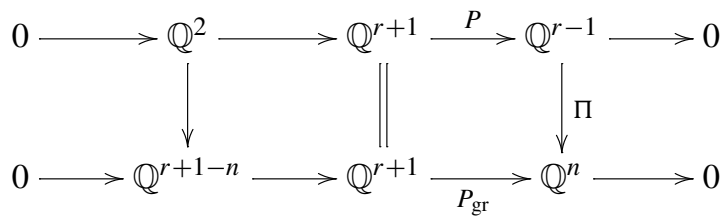

Note that $g_{1}$ equals $T^{\mu_{0}}$ times the pullback of the polynomial $h_{1}:=1+S_{1}+\cdots+$ $S_{n}$ under the homomorphism of tori $\mathbb{T}^{r} \rightarrow \mathbb{T}^{n}$ defined by $P_{\mathrm{gr}}$. The tropical variety of $V\left(h_{1}\right) \subseteq \mathbb{T}^{n}$ is the support of the fan $\Delta^{\prime}(n)$, and thus we have

$$
\operatorname{trop}\left(Y \cap T_{Z}\right)=\Pi^{-1}\left(\operatorname{trop}\left(V\left(h_{1}\right)\right)\right)=\Pi^{-1}\left(\left|\Delta^{\prime}(n)\right|\right) .
$$

We endow $\operatorname{trop}\left(Y \cap T_{Z}\right)$ with the natural fan structure lifting $\Delta^{\prime}(n)$; note that the cones are in general not pointed. By definition the weak tropical resolution $Y^{\prime}$ of $Y$ is the closure of $Y \cap T_{Z}$ in the toric variety $Z^{\prime}$ with the coarsest common refinement $\Sigma^{\prime}:=\Sigma \sqcap \operatorname{trop}\left(Y \cap T_{Z}\right)$ as its fan.

In the third step, we pass to $Y^{\prime}{ }_{0}^{\prime} T_{Y^{\prime}}$, where $T_{Y^{\prime}}$ is the kernel of the homomorphism of tori $T_{Z} \rightarrow \mathbb{T}^{n}$ defined by $\Pi$. By Construction 5.4 the quotient $Y_{0}^{\prime} T_{Y^{\prime}}$ is the closure of the image of $Y \cap T_{Z}$ under $T_{Z} \rightarrow \mathbb{T}^{n}$ in the toric variety $Z_{0}^{\prime \prime} T_{Y^{\prime}}$ associated to the describing fan in $\mathbb{Z}^{n}$ having as maximal cones the rays $\Pi(\varrho)$, where $\varrho$ runs through the rays of $\Sigma^{\prime}$.

Chaim. For every ray $\varrho \in \Sigma^{\prime}$, there is a facet of cone $\left(e_{0}, \ldots, e_{n-1}\right)$ containing the image $b:=\Pi(\varrho) \in \mathbb{Q}^{n}$. 
Indeed, since every cone of $\operatorname{trop}\left(Y \cap T_{Z}\right)$ is saturated with respect to $\Pi$, we have $\Pi(\varrho)=\Pi(\sigma) \cap \delta$ for some $\sigma \in \Sigma$ and $\delta \in \Delta^{\prime}(n)$. The image $\Pi(\sigma)$ is a cone spanned by some $e_{i}$ and some images $v_{j}:=\Pi\left(v_{j}\right)$, where $v_{j}$ are the primitive generators of the rays of $\Sigma$ different from columns $p_{i}$ of $P$. Proposition 4.1 yields the presentations

$$
v_{j}=\sum_{i=0}^{r-1} \alpha_{i j} p_{i} \quad \text { with certain } \alpha_{i j} \geq 0 .
$$

Hence, we obtain $v_{j} \in \operatorname{cone}\left(e_{0}, \ldots, e_{n-1}\right)$. Lemma 5.8 then shows that $\Pi(\varrho)$ lies in some facet of cone $\left(e_{0}, \ldots, e_{n-1}\right)$, and the claim is verified.

Finally, in the fourth step, we show that $Y_{0}^{\prime} T_{Y^{\prime}}$ is normal and has finitely generated Cox ring; by Proposition 5.7 this will complete the proof. First, note that we have the toric modification $Z_{0}^{\prime}{ }_{0}^{\prime} T_{Y^{\prime}} \rightarrow W$, where $W \subseteq \mathbb{P}_{n}$ is the open toric subset corresponding to the subfan $\Delta^{\prime}(n)$ of $\Delta(n)$. Moreover, $Y_{0}^{\prime \prime} T_{Y^{\prime}}$ is the proper transform under $Z_{0}^{\prime} T_{Y^{\prime}} \rightarrow W$ of the closure of $V\left(h_{1}\right) \subseteq \mathbb{T}^{n}$ in $W$. The claim just verified and Lemma 5.9 ensure that we may apply Proposition 3.3 and Corollary 3.4. In particular, we see that $Y_{0}^{\prime} / T_{Y^{\prime}}$ is normal with finitely generated Cox ring.

EXAmple 5.10. Consider the quadric $X=V\left(T_{0} T_{1}+\cdots+T_{6} T_{7}\right) \subseteq \mathbb{P}_{7}$ and the action of $\mathbb{K}^{*}$ on $\mathbb{P}_{7}$ given by

$$
t \cdot\left[x_{0}, \ldots, x_{7}\right]:=\left[t^{-3} x_{0}, t^{3} x_{1}, t^{-3} x_{2}, t^{3} x_{3}, t^{-2} x_{4}, t^{2} x_{5}, t^{-1} x_{6}, t x_{7}\right] .
$$

Theorem 1.2 and its proof do not apply to this case because only two weights $\zeta_{i}$ have minimal absolute value. The way through the weak toric resolution $Y^{\prime}$ as gone in the proof of Theorem 1.1 produces a quotient $Y_{0}^{\prime} T_{Y^{\prime}}$ embedded into the toric variety with fan obtained by subdividing $\Delta(3)$ at $(0,-1,-1)$.

\section{References}

[1] I. Arzhantsev, U. Derenthal, J. Hausen, and A. Laface, Cox rings, Cambridge Stud. Adv. Math., 144, Cambridge University Press, Cambridge, 2014.

[2] B. Bechtold, Factorially graded rings and Cox rings, J. Algebra 369 (2012), 351359.

[3] F. Berchtold and J. Hausen, Cox rings and combinatorics, Trans. Amer. Math. Soc. 359 (2007), no. 3, 1205-1252.

[4] D. Bourqui, La conjecture de Manin géométrique pour une famille de quadriques intrinsèques, Manuscripta Math. 135 (2011), no. 4, 1-41.

[5] A.-M. Castravet and J. Tevelev, $\bar{M}_{0, n}$ is not a Mori dream space, Duke Math. J. (to appear), arXiv:1311.7673.

[6] A. Craw and D. Maclagan, Fiber fans and toric quotients, Discrete Comput. Geom. 37 (2007), no. 2, 251-266.

[7] D. A. Cox, The homogeneous coordinate ring of a toric variety, J. Algebraic Geom. 4 (1995), no. 1, 17-50.

[8] I. V. Dolgachev and Y. Hu, Variation of geometric invariant theory quotients, Inst. Hautes Études Sci. Publ. Math. 87 (1998), 5-56, With an appendix by Nicolas Ressayre. 
[9] A. Gibney and D. Maclagan, Equations for Chow and Hilbert quotients, Algebra Number Theory 4 (2010), no. 7, 855-885.

[10] W. Gubler, A guide to tropicalizations, Algebraic and combinatorial aspects of tropical geometry, Contemp. Math., 589, pp. 125-189, Amer. Math. Soc., Providence, RI, 2013.

[11] S. Herrmann and M. Joswig, Totally splittable polytopes, Discrete Comput. Geom. 44 (2010), no. 1, 149-166.

[12] S. Herrmann, On the facets of the secondary polytope, J. Combin. Theory Ser. A 118 (2011), no. 2, 425-447.

[13] Y. Hu, Topological aspects of Chow quotients, J. Differential Geom. 69 (2005), no. 3, 399-440.

[14] Y. Hu and S. Keel, Mori dream spaces and GIT. Dedicated to William Fulton on the occasion of his 60th birthday, Michigan Math. J. 48 (2000), 331-348.

[15] M. M. Kapranov, Chow quotients of Grassmannians. I, I. M. Gel'fand seminar, Adv. Soviet Math., 16, Part 2, pp. 29-110, Amer. Math. Soc., Providence, RI, 1993.

[16] M. M. Kapranov, B. Sturmfels, and A. V. Zelevinsky, Quotients of toric varieties, Math. Ann. 290 (1991), no. 4, 643-655.

[17] J. Hausen, Cox rings and combinatorics. II, Mosc. Math. J. 8 (2008), no. 4, 711-757.

[18] J. Hausen and H. Süß, The Cox ring of an algebraic variety with torus action, Adv. Math. 225 (2010), no. 2, 977-1012.

[19] D. Mumford, J. Fogarty, and F. Kirwan, Geometric invariant theory, Third edition, Ergebnisse der Mathematik und ihrer Grenzgebiete (2), 34, Springer-Verlag, Berlin, 1994.

[20] S. Okawa, On images of Mori dream spaces, preprint, arXiv:1104.1326.

[21] M. Thaddeus, Geometric invariant theory and flips, J. Amer. Math. Soc. 9 (1996), no. 3, 691-723.

[22] J. Tevelev, Compactifications of subvarieties of tori, Amer. J. Math. 129 (2007), no. 4, 1087-1104.

H. Bäker

Mathematisches Institut

Universität Tübingen

Auf der Morgenstelle 10

72076 Tübingen

Germany

hendrik.baeker@uni-tuebingen.de

S. Keicher

Mathematisches Institut

Universität Tübingen

Auf der Morgenstelle 10

72076 Tübingen

Germany
J. Hausen

Mathematisches Institut

Universität Tübingen

Auf der Morgenstelle 10

72076 Tübingen

Germany

juergen.hausen@uni-tuebingen.de 